\title{
Aqueous humour outflow imaging: seeing is believing
}

\author{
Jong Yeon Lee ${ }^{1,2} \cdot$ Goichi Akiyama $^{1,3,4} \cdot$ Sindhu Saraswathy ${ }^{1} \cdot$ Xiaobin Xie $^{1,5} \cdot$ Xiaojing Pan $^{1,6} \cdot$ Young-Kwon Hong $^{7}$. \\ Alex S. Huang $\mathbb{1}^{1}$
}

Received: 14 August 2020 / Revised: 27 September 2020 / Accepted: 29 September 2020 / Published online: 15 October 2020

(c) The Author(s), under exclusive licence to The Royal College of Ophthalmologists 2020

\begin{abstract}
Elevated intraocular pressure (IOP) is the primary risk factor for blindness in glaucoma. IOP is determined by many factors including aqueous humour production and aqueous humour outflow (AHO), where AHO disturbance represents the primary cause of increased IOP. With the recent development of new IOP lowering drugs and Minimally Invasive Glaucoma Surgeries (MIGS), renewed interest has arisen in shedding light on not only how but where AHO is occurring for the trabecular/conventional, uveoscleral/unconventional, and subconjunctival outflow pathways. Historical studies critical to understanding outflow anatomy will be presented, leading to the development of modern imaging methods. New biological behaviours uncovered by modern imaging methods will be discussed with relevance to glaucoma therapies emphasized.
\end{abstract}

\section{Introduction}

Derangements in aqueous humour outflow (AHO) lead to elevated intraocular pressure (IOP) and can result in permanent vision loss from glaucomatous optic neuropathy. Elevated IOP is one of many glaucoma risk factors. Because it is the only modifiable risk factor, IOP reduction is the only available treatment.

IOP has been historically modelled by the Goldmann Equation: IOP $=($ Fin-Fout $) \mathrm{C}+\mathrm{EVP}[$ Fin $=$ aqueous production, Fout $=$ unconventional or uveoscleral outflow,

Alex S. Huang

Ahuang@Doheny.org

1 Doheny Eye Institute and Stein Eye Institute, Department of Ophthalmology, David Geffen School of Medicine, University of California, Los Angeles, California, USA

2 Department of Ophthalmology, Gachon University, College of Medicine, Gil Medical Center, Incheon, Korea

3 Jikei School of Medicine, Tokyo, Japan

4 Visual Research Project, Tokyo Metropolitan Institute of Medical Science, Tokyo, Japan

5 Eye Hospital of China Academy of Chinese Medical Sciences, Beijing, China

6 Qindao Eye Hospital of Shandong First Medical University, Shandong Eye Institute, Qindao, China

7 Department of Surgery, Keck School of Medicine, University of Southern California, Los Angeles, California, USA
$\mathrm{C}=$ conventional or trabecular outflow facility, and EVP = episcleral venous pressure] [1]. This relationship was developed from considerable work involving the study of aqueous humour production and outflow through multiple pathways. This equation has been instrumental in understanding ocular hypertension and how medicines or surgeries impact IOP [2]. However, this equation is an abstract concept that reduces AHO to a handful of numbers. While quantitation is key, a picture is still worth a thousand words. Seeing how aqueous moves in the context of the entire eye is important and holds promise for better understanding fundamental biology as well as uncovering new treatments.

Given the recent advent of Minimally Invasive Glaucoma Surgeries (MIGS; most of which target the conventional/trabecular pathway) and new glaucoma IOP lowering drugs that also target the trabecular pathways, there is significant interest in understanding both how [see Goldmann equation] and where aqueous humour flows. Based upon our current fundamental understanding, we know that this will involve the (a) conventional/trabecular and (b) unconventional/uveoscleral outflow pathways. Furthermore, during bleb-forming glaucoma surgeries (trabeculectomies, glaucoma drainage devices, and new subconjunctival MIGS), aqueous can also be shunted to a third route - the subconjunctival pathway. Thus, this review will delve into new AHO concepts with a focus on imaging where the fluid flows and on how this is relevant for glaucoma therapeutics. 


\section{The trabecular/conventional pathway}

\section{Discovery of trabecular outflow anatomy}

In 1830 Friedrich S. Schlemm discovered a circular canal at the junction of the sclera and cornea [3]. Many researchers investigated the role of the canal in terms of passing aqueous humour. The connections between the trabecular meshwork (TM) and Schlemm's canal (SC) were found by injecting dye into the anterior chamber and microscopically examining the specimens $[4,5]$.

Then outflow past the TM and SC was established using neoprene casts [6, 7]. Casting agents were injected into the eye, allowed to polymerize, and then after removal or digestion of ocular tissue, three-dimensional representations with detailed anatomical connections of SC to scleral veins were obtained without time-consuming histologic sectioning [6].

From this work, an overall picture of trabecular/conventional AHO emerged. AHO starts in the anterior chamber, and the trabecular pathway accounts for $\sim 90 \%$ of AHO under physiologic conditions [8, 9]. The trabecular pathway consists of the TM, SC, collector channels (CC), an intrascleral venous plexus, aqueous veins, and finally episcleral veins. In general, this pathway can be divided into proximal and distal portions. The proximal portion extends from TM to SC. The distal portion includes CCs and beyond.

In glaucoma, elevated IOP is caused by increased AHO resistance and not by excessive aqueous humour secretion [5]. The majority of this research has focused on the trabecular/conventional pathways. The main view is that, in open-angle glaucoma, the primary site of outflow resistance is at the juxtacanalicular tissue between TM and the inner wall of SC [10]. Thus, research in this field (including outflow imaging methods) has focused on the beginning of the trabecular/conventional pathways with less attention paid to what happens to AHO behind the TM.

Microscopic imaging of the proximal pathways initially used electron microscopy plus various tracers [11, 12]. Electron microscopy showed detailed structural pathways where aqueous could pass through the basement membrane of SC endothelial cells, giant vacuoles, and intercellular routes. Also, various tracers such as gold particles or cationic ferritin were applied [13, 14]. Electron microscopy could capture the tracer appearance in outflow pathways and even showed a segmental distribution across SC endothelial cells [14].

Ultimately, while the above work was critical in establishing a fundamental conventional AHO understanding, this research was laborious and unsuitable for live human subjects. Thus, a more modern approach was necessary. Additionally, variable IOP lowering after trabecular bypass or ablation MIGS raised the question of maintaining proximal AHO pathway patency after surgery as well as distal AHO contributions to resistance.

\section{Modern trabecular outflow imaging methods}

With the introduction of trabecular MIGS and the new class of cytoskeletal relaxing drugs, interest has grown in trying to understand the entire conventional AHO pathways proximal, distal, and circumferentially around the eye. Because of this there has been renewed interest in developing new imaging methods, either structure- or flowbased.

Structure-based conventional outflow imaging means visualizing the physical pathways. Two-photon microscopy has shown the structure of intact TM (from uveal to juxtacanalicular portions) and has even imaged deep to SC and $\mathrm{CC}$ openings [15]. Two-photon microscopy visualizes tissue architecture by using two photons of near-infrared longer wavelength light to achieve greater tissue penetration. Also, two-photon microscopy can be performed without fixation. However, the clearest images still come from tissue wedges, including the TM, that have been excised from the eye. 3D micro-CT then allowed for imaging the proximal and distal pathways in intact eyes using radiographic slices which could be stitched back together to generate an in situ outflow cast. 3D micro-CT is the same as clinical CT but performed on a smaller scale with higher resolution. This method was vital in showing outflow structural changes in response to IOP elevation as well as in detailed study of CCs $[16,17]$. However, post-mortem eyes were still being studied with fixation required.

The introduction of optical coherence tomography (OCT) led to the ability to assess ocular tissue in vivo in live human subjects. Initially developed for evaluating the posterior segment, OCT eventually expanded to the anterior segment and became a way to non-invasively assess AHO pathway structures such as TM, SC, and CCs. Early ASOCT studies provided cross-sectional images of the TM and SC in vivo [18] Cross-sectional area of SC was reported to be reduced in glaucoma [18]. Acute IOP elevation significantly decreased SC cross sectional area in healthy subjects [19]. Dilatation of SC area was observed following glaucoma medications, including pilocarpine hydrochloride [20]. An increased SC area was correlated with IOP reduction after trabeculoplasty [21].

However, AS-OCT still has limitations. Not all lumens in the anterior segment are necessarily involved in AHO. For example, there are arteries, veins and lymphatics in the anterior segment, and they all appear as lumens. Therefore, while SC can be clearly determined based on location, sorting distal outflow pathways from other luminal pathways can be confusing. Then, analysis of conventional 
pathway lumens is difficult because, unlike posterior segment OCT, no reference function exists where the OCT device can track the image to place the OCT scan in the same location and in the same orientation over time or after experimental manipulation. Thus, two different scans of the conventional outflow pathway can appear different solely because the scans were placed in a slightly different locations or because the subject tilted their head in a different way. To overcome this, 3D reconstructions of AHO pathways have been developed using OCT volumes so that the same volume could be theoretically created no matter the direction of scan [22]. However, this process is still tremendously time-consuming due to the large number of images needed to achieve high resolution (small B-scan to B-scan distance) while traversing the entire distance around the limbus. Therefore, while current OCT structural methods are non-invasive and suitable for live humans, technological innovations such as better tracking for a reference function and further increased speed and depth of penetration are needed.

To complement structural methods, tracer-based methods were developed to visualize the actual flow of aqueous humour. Starting with post-mortem eyes, tracers such as fluorescent microspheres ranging from 0.2-20 microns in size or 0.01 micron quantum dots were introduced into the anterior chamber [23-29]. Because of their size, these tracers accumulated in the TM, and they could be microscopically visualized after exposing the inner surface of the TM. The key finding was that conventional AHO was segmental. However, these methods were still not suitable for living human subjects.

To study patients, Fellman and Grover developed the episcleral venous fluid wave approach [30]. This method involved imaging visible blanching of episcleral veins after delivery of intraocular perfusate during surgery. Presence of the episcleral fluid wave was associated with surgical success after Trabectome surgery [31]. However, challenges included introducing perfusate at very high and nonphysiologic pressures (routine for intraocular surgery to maintain a stable chamber) and because the method did not involve the appearance of a signal as opposed to looking for the loss of a signal, which is hard.

Canalography was another approach that involved realtime conventional AHO imaging using pharmaceutical grade fluorescein or ICG, same as in retinal vascular angiography [32-34]. Canalography took advantage of exposing SC during canaloplasty surgery where tracer could be directly injected into the exposed SC end or during the reversal step where the canaloplasty probe is normally exited from the eye. During the reversal step, surgeons normally push out viscoelastic to expand SC, and for the purposes of outflow imaging, tracer could be used instead. While generating a positive signal of what conventional

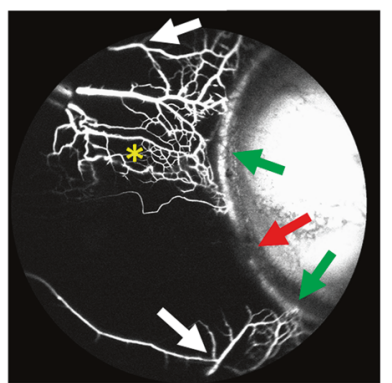

Fig. 1 Segmental aqueous humour outflow. ICG aqueous angiography was performed in an 82-year-old Caucasian man's left eye. The aqueous humour outflow pattern demonstrated regions of high-flow (green arrows) and low-flow (red arrow). Y-shaped episcleral and aqueous veins were seen (white-arrows) in addition to a network of vessels likely representing the intrascleral venous plexus (yellow asterisk) (color figure online).

AHO looked like, canalograms had two limitations. First, tracer was introduced into the SC and not the anterior chamber. Thus, the final outflow pattern did not include the TM contribution, which is unquestionably known to impact IOP. Then, tracer injection was always non-physiologic as $\mathrm{SC}$ is normally an extremely low-pressure location. Last, as the reversal step took time to perform, outflow was not being assessed circumferential around the limbus simultaneously. If injecting directly into exposed SC, tracer naturally arrived first near the injection site before the other side of the eye. Thus, comparison of segmental outflow could be difficult.

This led to the development of aqueous angiography which involved introducing tracers in the anterior chamber at physiologic pressures (Fig. 1). Aqueous angiography took advantage of FDA-approved starting materials. In a bedside-to-bench-to-bedside approach, the Spectralis HRA + OCT (Heidelberg Engineering, Germany) was brought into the lab. This device is FDA-approved to angiographically image blood flow after tracers such as fluorescein and indocyanine green (ICG) are intravenously injected. While not FDA-approved for intraocular use, these same tracers have been described as safe during off-label use as intraocular stains for membrane peels during pars plana vitrectomy or capsule staining for cataract surgery [35]. To further ease translating AHO imaging to patients, surgical tools (including side-port blades, anterior chamber maintainers, and surgical tubing) were utilized as well.

To perform aqueous angiography, constant-pressure gravity-driven tracer delivery was used. This started with post-mortem animal and human eyes in the laboratory showing again that conventional AHO was segmental [3638]. Then, the method was translated to the operating room for living non-human primates confirming segmental AHO [39]. Last, the method was adapted for human patients undergoing cataract surgery showing segmental AHO in live human subjects as well [40-42]. 
Recently, haemoglobin video imaging [43] has been developed which is a modernized quantitative approach to traditional observations of aqueous columns entering episcleral veins via aqueous veins. As aqueous is invisible to the naked eye, this method takes advantage of aqueous joining blood vessels where aqueous presence can be inferred next to the visible blood. The advantage is the noninvasive nature of this method. A limitation is that isolated aqueous veins situated away from episcleral veins, that only contain invisible aqueous, still cannot be visualized. Furthermore, appreciation of the aqueous column requires high magnification of these small intersections such that global visualization of overall outflow patterns is harder to achieve.

\section{New trabecular outflow concepts}

Segmental AHO was the primary discovery of new AHO research methods. Tracer based studies showed that outflow was not uniform throughout the TM [11, 13, 23-29]. TM tracer accumulation could be high or low, representing high- or low-flow regions. Percentage-effective filtration length could be calculated across the eye [24]. Regions of high- and low-flow TM could be isolated and studied showing fundamental biological differences in extracellular matrix and pro-fibrotic protein expression [27, 28, 44].

Aqueous angiography confirmed conventional segmental AHO in living non-human primate and human eyes [39-42] (Fig. 1). Using fluorescein aqueous angiography a limbalbased line scan method in healthy human subjects showed that $\sim 45 \%$ of the angiographic outflow was nasal while $\sim 23$ / 23/9\% was superior/inferior/temporal, respectively [41]. Melding clinical and basic science methods, simultaneous aqueous angiography using (a) fluorescent dextrans and (b) intracameral fluorescent microspheres showed that tracer trapping in the TM matched externally imaged aqueous angiographic outflow patterns [44]. This was important as similar results using various methods reassured the outcomes.

Currently, mechanisms governing segmental conventional AHO are unknown. Options include regulation at the level of the TM. This was suggested by the finding that bead-based tracer TM trapping patterns matched externally observed aqueous angiography aqueous outflow patterns [44]. Alternatively, segmental outflow patterns could arise due to more distal biological changes at CCs or aqueous/ episcleral veins. Each of these structures and pathways contain contractile elements, and local distal regulation could control segmental outflow patterns as well [45].

Pulsatile AHO was actually an old observation that was re-imaged with newer methods. Goldmann first described pulsatile flow, describing cyclic flow into aqueous veins that was synchronous with ocular pulse waves [46]. This pulsatile AHO was best observed at the clear fluid and blood junction where aqueous and episcleral veins met [47, 48] and what haemoglobin video imaging focuses on. Pulsatile AHO was believed to result from changes in choroidal volume that coincided with the cardiac cycle. It has been hypothesized that pulsatile AHO provides a passive mechanism to maintain short term IOP homoeostasis [49]. With respect to disease, pulsatile AHO has been noted to decrease with worsening glaucoma [47, 49]. Alternatively, IOP lowering medication has been shown to improve pulsatile aqueous outflow [50]. More recently, Phase-based OCT has shown pulse-dependent structural TM motion in enucleated non-human primate [51] and live human eyes [52]. This pulsatile motion synchronized with the digital pulse, further supporting a cardiac origin [50]. This was lastly confirmed with aqueous angiography in living nonhuman primates as tracer-based outflow imaging showed pulsatility rates $(120 \pm 52 \mathrm{bpm})$ which matched that of average non-human primates heart rates $(70-180 \mathrm{bpm})$ [39].

Dynamic AHO was a completely novel finding from aqueous outflow imaging research. Aqueous angiography was important for demonstrating dynamic AHO because it allowed real-time imaging in living subjects. Dynamic AHO was similar to pulsatile AHO in that AHO was not visually fixed. However, unlike pulsatile AHO (where the same AHO pattern simply increased and decreased in a cyclical fashion), in dynamic AHO, the entire pattern changed (Fig. 2). Low-flow regions could become highflow and vice-versa. Subjectively, it almost appeared as if the eye could increase or decrease overall outflow based on need. A few characteristics of dynamic AHO are important to highlight. First, these were infrequent and unpredictable events. In one study with $\sim 9$ min of video recording, only 16 dynamic events were seen [39]. Then, increasing and decreasing AHO could also be simultaneously observed in different locations of the same eye [53, 54]. Regarding mechanism, like segmental AHO, the control of dynamic AHO is unknown. Options also include local TM regulation where proximal outflow could be dynamically turned on or off. Studies using OCT and 3D-micro computed tomography (CT) have shown structural alterations to $\mathrm{CC}$, and this could also underlie dynamic AHO [17, 18]. Last, distal aqueous and episcleral veins are known to have contractile elements, and dynamic AHO may also be locally controlled by local vessel contraction and dilatation [45].

\section{Potential impact of trabecular outflow imaging on ocular hypertension treatment}

Conventional outflow imaging may help surgical and pharmacological approaches for IOP reduction. Pharmacologically, muscarinic agonists are known to lower IOP by contracting the longitudinal ciliary muscle to pull the scleral 

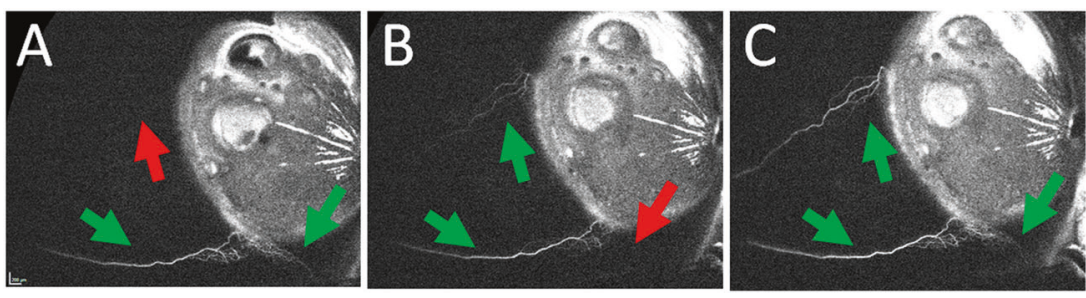

Fig. 2 Active aqueous humour outflow dynamic changes. ICG aqueous angiography was performed on the left eye of a 61-year-old Chinese female. a-c Shows dynamic aqueous humour outflow over

spur down and open the TM. However, a simple understanding of what muscarinic agents do to AHO patterns is actually not known. For example, overall outflow could minimally increase globally or focal areas could improve to a greater extent. If the latter is true, those TM areas could be isolated for study with critical features identified. These are the features that may be lost in ocular hypertension and the same features that may need to be recapitulated to treat it. This can lead to the identification of new drug-targets. Then, for distal outflow, cytoskeletal relaxing agents have been shown to dilate outflow pathways even after trabecular bypass using porcine eyes [55]. In this case, such drugs could be synergistically applied after trabecular bypass/ ablation to work in tandem for IOP lowering.

From a surgical perspective, trabecular MIGS are advantageous because they are fast and safe. However, IOP lowering magnitude can be variable, and this drove in part the desire to understand distal outflow better. For example, where the surgery is placed may matter. One question is whether trabecular MIGS should be targeted to high-flow regions or low-flow regions. Enhancing AHO in high-flow regions may be insufficient since high-flow regions already have good AHO. On the other hand, low-flow regions may have limited capability for AHO augmentation because of inadequate anatomy. Thus, conventional AHO imaging may be used to study surgical placement.

To address this question, a two-dye aqueous angiographic approach has been developed. First in post-mortem bovine eyes, ICG aqueous angiography, followed by fluorescein aqueous angiography showed similar segmental AHO patterns [37]. This established a model, and then in post-mortem human eyes sequential angiography was conducted to access the ability of trabecular bypass stents (iStent Inject, Glaukos Corporation) to improve AHO in regions of initially poor angiographic AHO [38]. ICG aqueous angiography established baseline outflow patterns. Trabecular bypass was performed in low-flow regions. Fluorescein aqueous angiography then showed $\sim 17$-fold improvement in angiographic signal compared to sham surgery eyes [38].

Moving to glaucoma patients, a similar study was performed [42]. ICG aqueous angiography established baseline
27 s (timing = A: time 0; B: 19 s later; C: $18 \mathrm{~s}$ after B). Green and red arrows show the appearance and disappearance of outflow pathways (color figure online).

outflow patterns in patients who were scheduled for cataract surgery and trabecular bypass. Trabecular bypass was performed in both low-flow and high-flow regions, and this was followed by fluorescein aqueous angiography. When surgery was performed in low-flow regions, transient to persistent angiographic outflow improvement was seen. This was termed "new recruitment". When placed in highflow regions, quicker angiographic improvement was seen. This was termed "earlier recruitment". In rare cases, no improvement occurred. IOP effects were unfortunately difficult to assess as the study only included 14 subjects. Furthermore, to stay consistent with the approved surgical method, two trabecular bypass stents were placed in each eye such that some eyes had both stents in low-flow regions, high-flow regions, or one in each. Thus, the conclusion was that trabecular bypass improved AHO many different ways, but it was still unclear which response is associated with the best IOP reduction. In the future, an improved study should utilize only one stent (either in low-flow or high-flow) with IOP compared in between. Ultimately, since the nasal side of the eye has more $\mathrm{CC}$, angiographic outflow is most common in the nasal area, and because most surgeons are accustomed to a temporal approach during cataract surgery, nasal placement is still recommended at this time until more data is acquired.

\section{The uveoscleral/unconventional pathway}

\section{Discovery of uveoscleral outflow anatomy}

The presence of an alternative outflow pathway was determined in the middle of the 20th century. After intraocular delivery, tracers accumulated in portions of the uvea, such as the choroid and ciliary body, and in posterior sclera. Some of the most quantitative and precise work was performed by Anders Bill in the 1960s using radiolabelled albumin.

Bill introduced radiolabelled albumin into the anterior chamber of multiple species, including non-human primates [56] and in a small number of human patients [9]. From this location, tracer could move through the conventional or 
unconventional pathways. During perfusion, the conventional pathways were queried when collecting perfusate from ocular surface veins [56] or blood from peripheral vasculature [57]. The main observation was that a significant portion of the introduced radiolabelled tracer could not be collected back from the conventional pathways. Thus, a second or unconventional pathway had to exist as another way for aqueous to exit the eye natively.

Tracer-based studies showed the route of the uveoscleral outflow pathway. Autoradiography could be performed on histological sections of the eye because the tracer was radiolabelled $[9,56]$. This showed that tracer accumulated in the sclera and uvea, nicely fitting the label of "uveoscleral" outflow. The uveoscleral pathway was thus proposed to start from the anterior chamber, flowing through the ciliary body in the angle (adjacent to the TM), past and into ciliary body clefts, into the supraciliary space, and then sclera or vortex veins.

Mechanistically, clefts that are naturally seen in normal ciliary body histological sections are important for uveoscleral outflow. Multiple drugs were shown to influence uveoscleral outflow as pilocarpine diminished the radio-labelled tracer uveoscleral spread while atropine enhanced it $[9,58]$. Ciliary muscle contraction or relaxation (by pilocarpine and atropine, respectively) abolished/ enhanced the presence of these clefts to enhance/attenuate uveoscleral outflow, respectively [59]. Furthermore, variation was seen in uveoscleral outflow. Older age was associated with less uveoscleral outflow; and some species such as non-human primates showed more while other species such as rabbits showed less [59]. When evaluating the ciliary body, younger age and species with more uveoscleral outflow demonstrated more ciliary body clefts [59]. This concept was clinically supported in humans because cases of trauma causing a different type of cleft (an artificial and large traumatic [60] ciliary body cleft) could lead to very low IOPs. Structurally, while UBM and OCT could not image native ciliary body clefts, secondary clefts (either from trauma or surgery [i.e., Cypass]) [61] could be visualized owing to their larger size.

While this review focuses on imaging, it is also important to point out that intraocular tracer studies are usually more difficult to perform in human patients owing to invasiveness, and this is particularly true for uveoscleral outflow. Thus, patient-based uveoscleral clinical studies typically used indirect methods based upon the Goldmann Equation: IOP $=$ (Fin-Fout)C + EVP $[1,59]$. As each of these variables could be measured in live humans, uveoscleral outflow (Fout) could be algebraically calculated. This type of work was important for showing that uveoscleral outflow was greater in younger individuals [8] and that prostaglandins increased uveoscleral outflow [62]. However, these indirect measures of uveoscleral outflow were notoriously variable, and could have low agreement compared to direct measures, even in the same study [63]. This was likely because each of the individual variables in the modified Goldmann equation were themselves indirectly determined and with their own set of assumptions.

From an imaging perspective, the closest attempt to realtime imaging of uveoscleral outflow in living subjects was performed by Butler et al. [64]. In this work, CT contrast medium (Amipaque; $\sim 350 \mathrm{MW}$ ) was introduced into the anterior chamber of living non-human primates during CT scan. Little signal was initially seen in the sclera (despite prolonged perfusion and after high IOP). However, after sacrifice of the non-human primate and with continued perfusion and CT scan, contrast signal could be eventually seen in the sclera. The authors interpreted this to mean that uveoscleral outflow could be better imaged after cardiac perfusion was stopped because native blood flow must have been washing away uveoscleral outflow tracer. However, another explanation exists and highlights just how difficult it is to image uveoscleral outflow. Since the tracer was placed in the anterior chamber, the tracer could have moved through the conventional pathway vessels and simply leaked out into the sclera after death, meaning that this work really imaged conventional AHO. Definite real-time uveoscleral outflow imaging in a living subject is thus still evasive.

\section{Modern uveoscleral outflow imaging methods}

The history of uveoscleral outflow assessment shows that imaging it is very difficult. In fact, it has not been performed real-time in a living subject. Thus, for this review, the question becomes how to develop strategies to image uveoscleral outflow more easily. The challenges are multiple. Work by Johnson and colleagues already elegantly summarize the challenges regarding tracer choice (molecular weight and issue of tracer retention) as well as the need to distinguish advective fluid flow versus diffusion [65]. Here, we focus more on anatomical considerations regarding the unique challenges of trying to image uveoscleral outflow in the setting of the rest of the eye.

The conventional and unconventional pathways share multiple potential touchpoints. They both start in the same location: the anterior chamber. Once distal, both pathways are heavily involved with the sclera (the intrascleral venous plexus for trabecular outflow and the scleral portion of uveoscleral outflow). The primary component of aqueous humour is water, and it is important to remember that no vessel is $100 \%$ impermeable to water. Thus, trabecular outflow can seep into the sclera when it passed through the intrascleral venous plexus, aqueous veins, and episcleral veins to potentially mix with uveoscleral outflow. This possibility is exactly what questions the interpretations of Butler et al. [64]. 
Also, it is because of this idea that one can consider the pathways as being integrated. It becomes very difficult to uniquely image uveoscleral outflow when trabecular outflow starts in the same location and can join uveoscleral outflow.

Both pathways can also be located in the same region of the eye such that uveoscleral outflow imaging can be blocked. For example, both conventional trabecular outflow and uveoscleral outflow can reside nasally. Then uveoscleral outflow is situated deeper as the ciliary body is deeper in the eye compared to the scleral wall where the TM, SC and the rest of the conventional pathway sits. If tracers enter both pathways, conventional trabecular outflow imaging can arise above and thus block visualization of deeper uveoscleral outflow.

To distinguish and image uveoscleral outflow, unique characteristics are needed to develop potential strategies. Examples could include: outflow patterns and the temporal nature of outflow. First, conventional outflow has recently been shown to be segmental (see Trabecular Outflow section, above). There are regions of high and low trabecular flow. Therefore, to avoid the problem of co-incidental location, trabecular outflow imaging could be performed first to identify low-flow regions of the eye as a way to concentrate uveoscleral imaging efforts to areas where trabecular outflow won't block it.

Second, the time frame is different. As mentioned above, measurement of uveoscleral outflow can be variable (depending on method), but is generally felt to be slow [65]. This is why uveoscleral outflow studies often take a few hours. This is in contrast to trabecular outflow imaging (see aqueous angiography) which occurs on the order of minutes.

Putting this all together, there are a few general strategies for developing a uveoscleral outflow imaging method. Conventional outflow imaging should be performed first to identify low-flow regions. Second, larger molecular weight tracers are advantageous because they are less likely to leak out of the conventional pathways into sclera. Third, observers must be patient as early signal is unlikely uveoscleral outflow while later signal elevates the ratio of uveoscleral compared to that of trabecular outflow. Last, consider pushing the system. This means overloading uveoscleral outflow. Native ciliary body clefts are important for uveoscleral outflow, and traumatic cyclodialysis clefts are just larger and more dramatic variants known to significantly lower IOP [60]. Surgical cyclodialysis clefts, such as the Cypass, are controlled versions that can also lower IOP. Thus, making artificial but large surgical clefts in regions of baseline low trabecular outflow may further improve local uveoscleral outflow visualization. Ultimately, imaging uveoscleral outflow is difficult and all of the above steps have not yet been attempted. Regardless of the exact method, uveoscleral outflow imaging will require careful validation because, as stated above, tracers introduced into the anterior chamber enter both the conventional and unconventional pathways and because these pathways merge around the sclera.

\section{New uveoscleral outflow concepts}

While real-time uveoscleral outflow imaging does not exist, other experimental systems have raised the new concept of uveolymphatic outflow [66]. This idea arose from immunofluorescence studies that challenged the traditional notion that intraocular lymphatics do not exist. Specifically, luminal pathways have been identified in the anterior uvea which express classical lymphatic molecular markers in certain species [66]. Furthermore, intraocular fluorescent tracer application led to tracer accumulation in local head and neck lymph nodes, suggesting an intraocular lymphatic route. This was enhanced by prostaglandins, suggesting that manipulation of the uveoscleral outflow pathway led to enhanced intraocular lymphatic tracer delivery [67]. However, as described above, no pathway is fully impermeable. The above intracameral tracer introduction could mean that tracer entered the conventional pathways and leaked out. If entering the subconjunctival space, subconjunctival lymphatics (see below Subconjunctival Outflow section) could have drained these tracers to local lymphatics and lymph nodes. Nevertheless, the old idea that central nervous tissue (and by extension the intraocular space) does not contain lymphatics is now known to be outdated. Lymphatics have been discovered in the uvea and even the meninges of the brain [68]. A glymphatic system that moves extracellular fluid paravascular from arteries to veins has also been found in the brain [69] and eye [70] and is under heavy investigation as related to sleep and neurodegenerative diseases, such as Alzheimer's disease. Therefore, more work is necessary to understand the importance of potential intraocular lymphatics and how this may be related to IOP.

\section{Potential impact of uveoscleral outflow imaging on ocular hypertension treatment}

While the benefit of clinical uveoscleral outflow imaging for IOP treatment is limited, because such imaging does not exist, there is potential for guiding treatment for both low and high IOP. For the most part, current examples use structural imaging methods.

For hypotony, knowing the location of a cleft causing increased uveoscleral outflow is beneficial for targeting surgical repair. Simple clefts can be seen by gonioscopy. OCT and UBM can provide an imaging alternative. However, if the cleft is small or with complex anatomy (that may occur in trauma), observation or 2D imaging can fail to identify the location. Alternatively, endoscopic imaging can 
be used as an examination tool to find and assist in the repair of small clefts [71]. Tracer studies have also been tested [72]. However, the goal was not to see the literal cleft. Instead, a small amount of trypan blue was placed in the centre of the anterior chamber. In normal circumstances, this dollop would evenly expand outward towards the limbus. In the presence of a cleft, the tracer instead preferentially and focally flowed toward the opening, giving a clue as to its location. Ultimately, while the above is useful, direct and real-time imaging of the cleft itself using tracers should still be more definitive.

For high IOP, uveoscleral MIGS have been developed (e.g., Cypass [Alcon]) or are under development (e.g., iStent Supra [Glaukos Corporation] or Miniject [iStar Medical]) to create controlled traumatic cyclodialysis clefts. Theoretically, such approaches have great potential because uveoscleral outflow avoids episcleral venous pressure which can act as an IOP reduction floor. The only FDA-approved uveoscleral MIGS was the Cypass (Alcon). Available for a limited time, Cypass failed due to corneal endothelial cell loss. However, lessons could be drawn from IOP responses as well. Clinically, Cypass lowered IOP, but failures were seen and anecdotally could be explosive with very high IOPs [73]. This was reminiscent of what is known regarding traumatic ciliary body clefts where IOP is initially low due to increased uveoscleral outflow but then can become very high if the cleft self-seals and the increased uveoscleral outflow goes away. In this case, underlying damage to the trabecular pathways is exposed. Structural anterior-segment OCT imaging has been performed for these surgeries, showing surgical "lakes" or clefts [61]. These are the same clefts that we proposed to create in low-flow trabecular outflow regions to assist in uveoscleral outflow imaging. In the future, structural OCT studies could be used to determine the relationship between IOP reduction and the surgical cleft size for uveoscleral MIGS. This could improve our understanding of this outflow pathway and help identify patients where the surgery may be structurally failing due to progressive shrinking of the surgical clefts.

\section{The subconjunctival outflow pathways}

\section{Discovery of subconjunctival outflow anatomy}

Subconjunctival outflow refers to clearance of material from the subconjunctival space. Thus, it is native to the eye and happens all of the time. The subconjunctival space is essentially a potential space that can be opened under pathologic conditions (chemosis from fluid overload in hospital patients or from conjunctivitis in eye clinic patients) or during the treatment of eye diseases (subconjunctival injection of antibiotics and steroids at the end of ocular surgery or in a bleb that is purposely created during glaucoma surgery). However, these examples demonstrate how subconjunctival outflow is different from trabecular/conventional and uveoscleral/unconventional outflow. The latter two are involved in the normal physiology of aqueous humour homoeostasis. Derangements therein lead to elevated IOP, and direct manipulation can lower (e.g., pilocarpine) or raise IOP (e.g., atropine). Subconjunctival outflow is involved in the normal passage of aqueous from the eye in the sense of clearing the fluid that seeps out from trabecular and uveoscleral outflow pathways into the space under the conjunctiva. No evidence yet exists to suggest that subconjunctival outflow directly impacts normal physiologic IOP. However, because of potential impact on glaucoma surgery and drug delivery, subconjunctival outflow may be leveraged to improve eye care.

The presence of subconjunctival outflow is implied in chemosis. In certain pathologic conditions such as fluid overload, or during ocular surface inflammation/infection, the conjunctiva raises with fluid filling the subconjunctival space. However, chemosis resolves when the underlying disorder is treated. Similarly, at the end of glaucoma surgery, drugs can be injected under the conjunctiva to create a drug delivery bleb. However, all surgeons know that by the next day, the bleb is gone. Thus, subconjunctival fluid is somehow draining. This has been studied using tracer-based methods (Fig. 3).

Two common systems to study tracer egress from under the conjunctiva have been via direct subconjunctival tracer injection or by the study of glaucoma surgical blebs. Early observations included documenting the natural resolution of spontaneous subconjunctival haemorrhages. Blood would resolve and form sausage-like structures [74], suggesting an outflow pathway. Scleral tattooing gone awry could also show distinct patterns [74]. Subconjunctival injection of ICG performed in live humans demonstrated patterns that were unlike typical Y-shaped episcleral veins [74, 75]. Quantitative assessment of subconjunctival drug depot clearance was substantially higher in living as opposed to post-mortem eyes, suggesting an active mechanism [76].

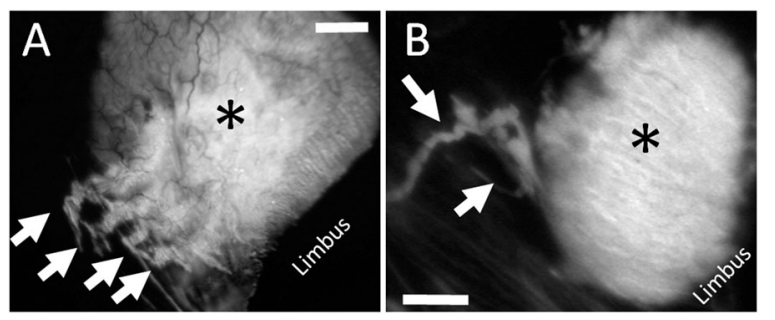

Fig. 3 Subconjunctival bleb-related outflow pathways. Fixable and fluorescent dextrans $(500 \mathrm{kD} ; 2.5 \mathrm{mg} / \mathrm{ml})$ were injected under the conjunctiva of post-mortem (a) human and (b) porcine eyes to create blebs (black asterisks). Bleb-related outflow pathways were seen (white arrows). Scale bars: $1 \mathrm{~mm}$. 
Together, these results demonstrated a formal process for fluid removal from the subconjunctival space and raised the possibility of using lymphatics.

Glaucoma surgical subconjunctival blebs are created by making a direct communication between the anterior chamber and subconjunctival space for the purpose of lowering IOP. This has led to the long-standing question of how aqueous flowing into glaucoma surgical blebs subsequently exits. The route also has to be a low-resistance pathway or else glaucoma surgery could not lower IOP. Early research suggested aqueous humour exited mainly through a transconjunctival route in trabeculectomy blebs. Kronfield confirmed this by showing that the surface of filtering blebs had elevated levels of ascorbic acid, a small molecule known to be enriched in aqueous humour [77]. Functioning filtering blebs were also documented on light and electron microscopy to have loosely arranged subepithelial connective tissue with clear spaces [78, 79]. Ultrastructural study of filtration blebs showed that these clear spaces were microcysts.

However, all glaucoma surgeons also know that trabeculectomy blebs can be highly varied and that tall/thin/cystic blebs that maybe weeping aqueous through the conjunctiva are not ideal. Furthermore, glaucoma surgeons also know that, clinically, we can often see trabeculectomy patients with good IOP control even when there is no obvious bleb. These observations motivated researchers to search for other bleb-related outflow pathways. Teng et al. proposed resorption through degenerative veins in the bleb [80]. Benedikt introduced fluorescein into the anterior chamber after glaucoma surgery and witnessed multiple potential routes for aqueous outflow including newly incorporated conjunctival veins and possibly lymphatics [81].

Lymphatics are a one-way non-circulatory system that are responsible for extracellular fluid homoeostasis, fat absorption, and immune surveillance [82]. Long known to exist in the conjunctiva, this system involves a vast network of thin-walled capillaries, large collecting vessels, and secondary lymphoid tissue. They respond to increased interstitial pressure by widening their lumina to allow extracellular fluid in. Lymph then returns to the venous circulation via the thoracic duct. Given their known role in regulating extracellular fluid, it made sense to consider that lymphatics could clear the subconjunctival space.

More recently, subconjunctival MIGS have become available (Xen from Allergan) or are under advanced stages of development (Preserflo from Santen), and this has further driven the desire to better understand subconjunctival outflow. Substantial work was performed by Dao Yi Yu's research team during Xen development [83, 84]. Subconjunctival injection of trypan blue led to irregular pathways arising from blebs in rabbits and non-human primates [83]. After Xen surgery, introduction of fluorescein that delineated surgical blebs also showed irregular pathways arising off blebs [83]. In trabeculectomy patients, better IOP lowering was seen in cases where the blebs also showed these types of pathways [85]. Overall, these pathways were hypothesized to be lymphatic. However, this was presumed based on the known presence of conjunctival lymphatics [86], the fact that lymphatics should drain extracellular space, and because the pathways off the blebs did not look like veins. The actual pathways themselves were never isolated for study to determine the exact identity.

\section{Modern subconjunctival outflow imaging methods}

Tracer studies have been critical in demonstrating subconjunctival outflow, but these studies alone could not provide the identity of the pathways. Thus, multi-modal approaches were taken in porcine eyes to combine tracer studies with other methods [87]. After injecting tracers under the conjunctiva to create blebs and outflow pathways, concurrent cross-sectional OCT demonstrated that these pathways were distinct and luminal [87]. OCT longitudinally along these pathways further demonstrated the presence of semi-lunar valves orientated in the direction of flow [87]. Two-photon microscopy of the pathways showed blind-end tips [87]. These last two observations were critical because lymphatics have valves and blind-end tips. In fact, the valves are so obvious that investigators can find these pathways using OCT alone on the ocular surface, just by searching for these structures [84].

Fixable and fluorescent dextrans were also used to create subconjunctival blebs and outflow pathways for multimodal study combining tracer-based studies with immunofluorescence [87]. These tracers were chosen because they could be angiographically visualized using fluorescence imaging, and after fixation they could be trapped to the outflow luminal wall. Because of this, histological sectioning could be guaranteed to find the exact same pathway that was initially seen on outflow imaging. Then, immunofluorescence for lymphatic markers (Prox-1 and podoplanin) showed co-localization with the subconjunctival outflow pathways while blood vessel markers (CD31) did not [87]. This provided molecular proof for lymphatic outflow, complemented the above structural evidence, and together finally confirmed the previously raised hypothesis that lymphatics drain blebs.

To further study subconjunctival outflow, genetic rodent models now represent a powerful new tool. Prox-1-EGFP BAC has been incorporated into mice [88] and rats [89] to generate animals with natively fluorescent lymphatics. A Prox-1-tdTomato transgenic line is also available [90]. Outside of the eye, these rodents have been used to visualize lymphatics in transplantation models [88] and to study 


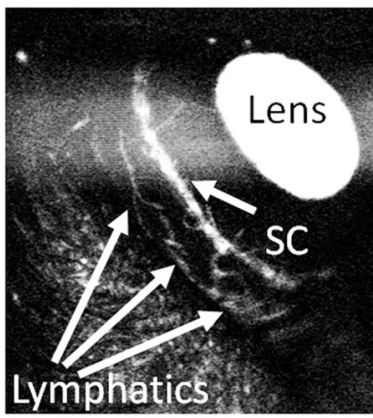

Fig. 4 In vivo imaging of subconjunctival lymphatics. A fluorescent lymphatic reporter mouse was imaged that expressed GFP under a PROX-1 promoter. PROX-1 is expressed in lymphatics, lens epithelium, and Schlemm's canal (SC). In vivo imaging in a live anaesthetised mouse clearly showed subconjunctival lymphatics.

lymphangiogenesis as a way to combat post-surgical lymphedema [91]. In the eye, subconjunctival lymphatics are visible in flat-mount and even during in vivo imaging (Fig. 4). Using these mice, lymphatic development and manipulation can be studied.

\section{New subconjunctival outflow concepts}

Subconjunctival lymphatics are not evenly distributed across the eye. This was first seen in the lymphatic reporter mice, where post-natal lymphatic development was studied, and lymphatics were found to develop from a nasal origin and then wrap around to the temporal side of the eye [92]. Thus, in adult mice, an 4 -fold increased number of lymphatic sprouts were seen on the nasal compared to the temporal side of the eye [92]. Functionally, greater nasal lymphatic outflow was seen as subconjunctival tracers cleared on the nasal side of the eye more efficiently than on the temporal side of the eye [92]. This mouse data agreed with porcine results that showed a $\sim 2$-fold increase in the number of outflow pathways seen off tracer-created nasal blebs compared to temporal ones [87]. Together, these results showed a subconjunctival lymphatic asymmetry across the anterior eye that could be leveraged for treatment of ocular diseases.

In addition to a native heterogeneous distribution of subconjunctival lymphatics, pharmacological manipulation of lymphatics may also be another option to create further non-homogeneity. Multiple agents have been shown to increase lymphatic presence in the body. Vascular endothelial growth factor type $\mathrm{C}$ (VEGFC) is the primary endogenous lymphatic vessel growth factor [93]. Alternatively, VEGFA promotes both blood vessel and lymphatic growth. Known mutations for VEGFC (C156S) are further very specific for growing only lymphatics [94]. 9-cis Retinoic acid can also promote lymphatic growth and has been shown to ameliorate lymphedema in a combined radiation and surgical injury rodent lymphedema model [91]. Alternatively, it has been hypothesized that anti-metabolites used during glaucoma surgery (mitomycin$\mathrm{C}$ [MMC] and 5-fluorouracil [5FU]) may damage lymphatics [95]. After glaucoma surgery, failed trabeculectomy blebs are devoid of lymphatics while peri-bleb tissue shows an intermediate presence with normal conjunctiva demonstrating a robust lymphatic network [96]. Therefore, future concepts can include drug-induced lymphatic manipulation (for either more or less) as a potential way to treat eye diseases.

\section{Potential impact of subconjunctival outflow imaging on ocular hypertension treatment}

Subconjunctival outflow may be leveraged to improve blebbased glaucoma surgeries or to improve drug delivery which can enhance the treatment of nearly all eye diseases. For glaucoma surgery, the issue of blebs is that they sometimes fail. This can be the case even if a bleb is visually present. As mentioned above, failed blebs have been shown to be devoid of subconjunctival lymphatics [96]. It can be hypothesized that anti-metabolite use during glaucoma surgery used to prevent bleb scarring may also damage lymphatics such that aqueous cannot exit the bleb in a kind of blind cul-du-sac. In this case, subconjunctival lymphatic enhancement (either by placing surgeries in regions enriched with subconjunctival lymphatics or via drug manipulation such as by VEGFC) could improve blebbased IOP lowering.

Alternatively, diminishing lymphatics may be beneficial for subconjunctival drug delivery. Drug delivery strategies are advantageous because poor eyedrop adherence and persistence are known problems. Intravitreal injections are common but risks include pain, infection, and retinal detachment. Subconjunctival drug injections would be ideal as they are more superficial and easier to administer [97]. However, drug penetration into the eye is a challenge. Subconjunctival drug has to get past the sclera as well as the choroid and retinal pigment epithelium to become truly intraocular. Currently, medium sized proteins have been shown to be capable of getting past these barriers [97]. However, any agent delivered under the conjunctiva is also likely to be drained away by subconjunctival lymphatics such that a depot could not persist. In this case, strategies to inject drugs where there are fewer lymphatics or to locally diminish subconjunctival lymphatics may work to enhance the subconjunctival space as a drug-delivery target.

Ultimately, future work is necessary to establish whether lymphatic manipulation can either improve glaucoma surgical bleb function or subconjunctival drug delivery into the eye. More imaging methods for tracer flow or structural evaluation of bleb-related pathways will be needed in live patients to assess the success of these strategies [98]. 


\section{Conclusion}

In the last decade, the advent of new drugs and surgeries to lower IOP and treat glaucoma has re-invigorated the desire to understand how and where AHO is occurring in the conventional, unconventional, and subconjunctival outflow pathways. Current imaging methods are at varying stages of development with conventional outflow imaging being the most advanced, followed by subconjunctival outflow imaging. Uveoscleral outflow is the most difficult to image although potential strategies are proposed. Already, we know that each route has unique biology that can be leveraged. For trabecular/conventional outflow, ocular hypertension may be an expansion of normally low-flow segmental areas and additional research can lead to a better understanding about why IOP increases in disease and how to lower it by modified drug and surgical approaches. For uveoscleral/unconventional outflow, better imaging may help treat both hypotony and ocular hypertension. For high IOP, uveoscleral surgical approaches still have the benefit of avoiding episcleral venous pressure for very strong IOP reduction so that this demands the development of imaging tools to study these surgeries. For subconjunctival outflow, lymphatic manipulation in either direction has potential benefit. Bleb-related surgeries (those surgeries reserved for the most advanced glaucoma) may be able to be enhanced by improving lymphatic outflow. Improving drug delivery through the subconjunctival route is also important as it can improve treatment of all drugrequiring eye diseases. In the end, with better visualization of how and where AHO is occurring, improved fundamental understanding of ocular hypertension in glaucoma may be achieved and current treatments may be able to be improved while opening avenues to innovate new ones.

Acknowledgements This work was supported by China Scholarship Council Grant (\#201808110001), Capital Characteristic Clinic Project of Beijing (Z18110000171808) [XX]; NIH NEI R01EY030501 [ASH], R21EY026260 [YH]; Qindao Benefit People Demonstration Guide Special Project 20-3-4-39-nsh [XP]; Glaucoma Research Foundation Shaffer Grant [ASH]; Research to Prevent Blindness Career Development Award 2016 [ASH]; an unrestricted grant from Research to Prevent Blindness [UCLA]; Japan Society for the Promotion of Science (JP18K16972) [GA]. The sponsors or funding organizations had no role in this manuscript. ASH is a consultant for (Allergan, Santen, Gore, and Aerie), he has received research support from (Heidelberg Engineering, Glaukos, and Diagnosys), he has received speaker fees from (Heidelberg Engineering, Glaukos, and Santen). The remaining authors have no financial disclosures.

\section{Compliance with ethical standards}

Conflict of interest The authors declare that they have no conflict of interest.

Publisher's note Springer Nature remains neutral with regard to jurisdictional claims in published maps and institutional affiliations.

\section{References}

1. Brubaker RF. Goldmann's equation and clinical measures of aqueous dynamics. Exp Eye Res. 2004;78:633-7.

2. Alaghband P, Beltran-Agullo L, Galvis EA, Overby DR, Lim KS. Effect of phacoemulsification on facility of outflow. Br J Ophthalmol. 2018;102:1520-6.

3. Schlemm F. Bulbus oculi. In: Th. Chr. Fr. Enslin. Theoretischpraktisches Handbuch der Chirurgie, mit Einschluß der syphilitischen und Augen-Krankheiten. Berlin, Germany: Carl Gerold, Wien, 1830. pp 331-338.

4. Schwalbe G. Untersuchungen über die Lymphbahnen des Auges und ihre Begrenzungen. Arch für mikroskopische Anat. $1870 ; 6: 261-362$.

5. Leber T. Studien über den Flüssigkeitswechsel im Auge. Albrecht von Graefes Arch für Ophthalmologie. 1873;19:87-185.

6. Ashton N. Anatomical study of Schlemm's canal and aqueous veins by means of Neoprene casts: Part I. Aqueous veins. Br J Ophthalmol. 1951;35:291.

7. Ashton N, Smith R. Anatomical study of Schlemm's canal and aqueous veins by means of Neoprene casts: III. Arterial relations of Schlemm's canal. Br J Ophthalmol. 1953;37:577.

8. Toris CB, Yablonski ME, Wang Y-L, Camras CB. Aqueous humor dynamics in the aging human eye. Am J Ophthalmol. 1999;127:407-12.

9. Bill A, Phillips CI. Uveoscleral drainage of aqueous humour in human eyes. Exp Eye Res. 1971;12:275-81.

10. Overby DR, Stamer WD, Johnson M. The changing paradigm of outflow resistance generation: towards synergistic models of the JCT and inner wall endothelium. Exp Eye Res. 2009;88:656-70.

11. Inomata H, Bill A, Smelser GK. Aqueous humor pathways through the trabecular meshwork and into Schlemm's canal in the cynomolgus monkey (Macaca irus): an electron microscopic study. Am J Ophthalmol. 1972;73:760-89.

12. Ujiie K, Bill A. The drainage routes for aqueous humor in monkeys as revealed by scanning electron microscopy of corrosion casts. Scan Electron Microsc. 1984;(Pt 2):849-56.

13. Hann CR, Bahler CK, Johnson DH. Cationic ferritin and segmental flow through the trabecular meshwork. Invest Ophthalmol Vis Sci. 2005;46:1-7.

14. Sabanay I, Gabelt BT, Tian B, Kaufman PL, Geiger B. H-7 effects on the structure and fluid conductance of monkey trabecular meshwork. Arch Ophthalmol. 2000;118:955-62.

15. Huang AS, Gonzalez JM, Le PV, Heur M, Tan JC. Sources of structural autofluorescence in the human trabecular meshwork. Investigative Ophthalmol Vis Sci. 2013;54:4813-20.

16. Hann CR, Bentley MD, Vercnocke A, Ritman EL, Fautsch MP. Imaging the aqueous humor outflow pathway in human eyes by three-dimensional micro-computed tomography (3D micro-CT). Exp Eye Res. 2011;92:104-11.

17. Hann CR, Vercnocke AJ, Bentley MD, Jorgensen SM, Fautsch MP. Anatomic changes in Schlemm's canal and collector channels in normal and primary open-angle glaucoma eyes using low and high perfusion pressures. Invest Ophthalmol Vis Sci. 2014;55:5834-41.

18. Kagemann L, Wollstein G, Ishikawa H, Bilonick RA, Brennen PM, Folio LS, et al. Identification and assessment of Schlemm's canal by spectral-domain optical coherence tomography. Investigative Ophthalmol Vis Sci. 2010;51:4054-9.

19. Kagemann L, Wang B, Wollstein G, Ishikawa H, Nevins JE, Nadler Z, et al. IOP elevation reduces Schlemm's canal crosssectional area. Investigative Ophthalmol Vis Sci. 2014;55:1805-9.

20. Skaat A, Rosman MS, Chien JL, Mogil RS, Ren R, Liebmann JM, et al. Effect of pilocarpine hydrochloride on the Schlemm canal in 
healthy eyes and eyes with open-angle glaucoma. JAMA Ophthalmol. 2016;134:976-81.

21. Skaat A, Rosman MS, Chien JL, Ghassibi MP, Liebmann JM, Ritch R, et al. Microarchitecture of Schlemm canal before and after selective laser trabeculoplasty in enhanced depth imaging optical coherence tomography. J Glaucoma. 2017;26:361-6.

22. Huang AS, Belghith A, Dastiridou A, Chopra V, Zangwill LM, Weinreb RN. Automated circumferential construction of firstorder aqueous humor outflow pathways using spectraldomain optical coherence tomography. J Biomed Opt. 2017;22:066010.

23. Swaminathan SS, Oh DJ, Kang MH, Ren R, Jin R, Gong H, et al. Secreted protein acidic and rich in cysteine (SPARC)-null mice exhibit more uniform outflow. Invest Ophthalmol Vis Sci. 2013;54:2035-47.

24. Battista SA, Lu Z, Hofmann S, Freddo T, Overby DR, Gong H. Reduction of the available area for aqueous humor outflow and increase in meshwork herniations into collector channels following acute IOP elevation in bovine eyes. Invest Ophthalmol Vis Sci. 2008;49:5346-52.

25. Lu Z, Overby DR, Scott PA, Freddo TF, Gong H. The mechanism of increasing outflow facility by rho-kinase inhibition with Y27632 in bovine eyes. Exp Eye Res. 2008;86:271-81.

26. Vranka JA, Bradley JM, Yang YF, Keller KE, Acott TS. Mapping molecular differences and extracellular matrix gene expression in segmental outflow pathways of the human ocular trabecular meshwork. PLoS ONE. 2015;10:e122483.

27. Keller KE, Bradley JM, Vranka JA, Acott TS. Segmental versican expression in the trabecular meshwork and involvement in outflow facility. Invest Ophthalmol Vis Sci. 2011;52:5049-57.

28. Vranka JA, Acott TS. Pressure-induced expression changes in segmental flow regions of the human trabecular meshwork. Exp Eye Res. 2017;158:67-72.

29. Vranka JA, Staverosky JA, Raghunathan V, Acott TS. Elevated pressure influences relative distribution of segmental regions of the trabecular meshwork. Exp Eye Res. 2020;190:107888.

30. Fellman RL, Grover DS. Episcleral venous fluid wave: intraoperative evidence for patency of the conventional outflow system. J glaucoma. 2014;23:347-50.

31. Fellman RL, Feuer WJ, Grover DS. Episcleral venous fluid wave correlates with trabectome outcomes: intraoperative evaluation of the trabecular outflow pathway. Ophthalmology. 2015;122: 2385-91.e1.

32. Grieshaber MC. Ab externo Schlemm's canal surgery: viscocanalostomy and canaloplasty. Dev Ophthalmol. 2012;50:109-24.

33. Grieshaber MC, Pienaar A, Olivier J, Stegmann R. Clinical evaluation of the aqueous outflow system in primary open-angle glaucoma for canaloplasty. Invest Ophthalmol Vis Sci. 2010;51:1498-504.

34. Zeppa L, Ambrosone L, Guerra G, Fortunato M, Costagliola C. Using canalography to visualize the in vivo aqueous humor outflow conventional pathway in humans. JAMA Ophthalmol. 2014;132:1281.

35. Jacobs DS, Cox TA, Wagoner MD, Ariyasu RG, Karp CL, American Academy of $\mathrm{O}$. et al. Capsule staining as an adjunct to cataract surgery: a report from the American Academy of Ophthalmology. Ophthalmology. 2006;113:707-13.

36. Saraswathy S, Tan JC, Yu F, Francis BA, Hinton DR, Weinreb $\mathrm{RN}$, et al. Aqueous angiography: real-time and physiologic aqueous humor outflow imaging. PLoS ONE. 2016;11:e0147176.

37. Huang AS, Saraswathy S, Dastiridou A, Begian A, Legaspi H, Mohindroo $\mathrm{C}$, et al. Aqueous angiography with fluorescein and indocyanine green in bovine eyes. Transl Vis Sci Technol. 2016;5:5-

38. Huang AS, Saraswathy S, Dastiridou A, Begian A, Mohindroo C, Tan JC, et al. Aqueous angiography-mediated guidance of trabecular bypass improves angiographic outflow in human enucleated eyes. Investigative Ophthalmol Vis Sci. 2016;57:4558-65.

39. Huang AS, Li M, Yang D, Wang H, Wang N, Weinreb RN. Aqueous angiography in living nonhuman primates shows segmental, pulsatile, and dynamic angiographic aqueous humor outflow. Ophthalmology. 2017;124:793-803.

40. Huang AS, Camp A, Xu BY, Penteado RC, Weinreb RN. Aqueous angiography: aqueous humor outflow imaging in live human subjects. Ophthalmology 2017;124:1249-51.

41. Huang AS, Penteado RC, Saha SK, Do JL, Ngai P, Hu Z, et al. Fluorescein aqueous angiography in live normal human eyes. J Glaucoma. 2018;27:957-64.

42. Huang AS, Penteado RC, Papoyan V, Voskanyan L, Weinreb RN. Aqueous angiographic outflow improvement after trabecular microbypass in glaucoma patients. Ophthalmol Glaucoma. 2019;2:11-21.

43. Khatib TZ, Meyer PAR, Lusthaus J, Manyakin I, Mushtaq Y, Martin KR. Hemoglobin video imaging provides novel in vivo high-resolution imaging and quantification of human aqueous outflow in patients with glaucoma. Ophthalmol Glaucoma. 2019;2:327-35.

44. Saraswathy S, Bogarin T, Barron E, Francis BA, Tan JCH, Weinreb RN, et al. Segmental differences found in aqueous angiographic-determined high - and low-flow regions of human trabecular meshwork. Exp Eye Res. 2020;196:108064.

45. Gonzalez JM, Ko MK, Hong Y-K, Weigert R, Tan JC. Deep tissue analysis of distal aqueous drainage structures and contractile features. Sci Rep. 2017;7:1-20.

46. Goldmann H. Abfluss des kammerwassers beim menschen. Ophthalmologica 1946;111:146-52.

47. Ascher K. The aqueous veins*: I. Physiologic importance of the visible elimination of intraocular fluid. Am J Ophthalmol. 2018;192:xxix-liv.

48. Thomassen T, Perkins E, Dobree J. Aqueous veins in glaucomatous eyes. Br J Ophthalmol. 1950;34:221.

49. Johnstone M, Martin E, Jamil A. Pulsatile flow into the aqueous veins: manifestations in normal and glaucomatous eyes. Exp Eye Res. 2011;92:318-27.

50. Xin C, Wang RK, Song S, Shen T, Wen J, Martin E, et al. Aqueous outflow regulation: optical coherence tomography implicates pressure-dependent tissue motion. Exp Eye Res. 2017;158:171-86.

51. Hariri S, Johnstone M, Jiang Y, Padilla S, Zhou Z, Reif R, et al. Platform to investigate aqueous outflow system structure and pressure-dependent motion using high-resolution spectral domain optical coherence tomography. J Biomed Opt. 2014;19:106013.

52. Li P, Shen TT, Johnstone M, Wang RK. Pulsatile motion of the trabecular meshwork in healthy human subjects quantified by phase-sensitive optical coherence tomography. Biomed Opt Express. 2013;4:2051-65.

53. Huang AS, Francis BA, Weinreb RN. Structural and functional imaging of aqueous humour outflow: a review. Clin Exp Ophthalmol. 2018;46:158-68.

54. Xie X, Akiyama G, Bogarin T, Saraswathy S, Huang AS. Visual assessment of aqueous humor outflow. Asia Pac J Ophthalmol (Phila). 2019. https://doi.org/10.22608/APO.201911. e-pub ahead of print.

55. Waxman S, Wang C, Dang Y, Hong Y, Esfandiari H, Shah P, et al. Structure-function changes of the porcine distal outflow tract in response to nitric oxide. Invest Ophthalmol Vis Sci. 2018;59:4886-95.

56. Bill A. The aqueous humor drainage mechanism in the cynomolgus monkey (Macaca irus) with evidence for unconventional routes. Invest Ophthalmol. 1965;4:911-9.

57. Bill A. Conventional and uveo-scleral drainage of aqueous humour in the cynomolgus monkey (Macaca irus) at normal and high intraocular pressures. Exp Eye Res. 1966;5:45-54. 
58. Bill A. Effects of atropine and pilocarpine on aqueous humour dynamics in cynomolgus monkeys (Macaca irus). Exp Eye Res. 1967;6:120-5.

59. Huang AS, Weinreb RN Structure and Mechanism of Uveoscleral Outflow. In: Francis BA, Sarkisian SR, Tan JC, editors. Minimally Invasive Glaucoma Surgery. New York: Thieme; 2017. pp 25-33.

60. Wang Q, Thau A, Levin AV, Lee D. Ocular hypotony: a comprehensive review. Surv Ophthalmol. 2019;64:619-38.

61. Saheb H, Ianchulev T, Ahmed IIK. Optical coherence tomography of the suprachoroid after CyPass Micro-Stent implantation for the treatment of open-angle glaucoma. $\mathrm{Br} \mathrm{J}$ Ophthalmol. 2014;98:19-23.

62. Toris CB, Camras CB, Yablonski ME. Effects of PhXA41, a new prostaglandin F2 alpha analog, on aqueous humor dynamics in human eyes. Ophthalmology. 1993;100:1297-304.

63. Toris CB, Zhan GL, Wang YL, Zhao J, McLaughlin MA, Camras $\mathrm{CB}$, et al. Aqueous humor dynamics in monkeys with laserinduced glaucoma. J Ocul Pharm Ther. 2000;16:19-27.

64. Butler JM, Raviola G, Beers GJ, Carter AP. Computed tomography of aqueous humour outflow pathways. Exp Eye Res. 1984;39:709-19.

65. Johnson M, McLaren JW, Overby DR. Unconventional aqueous humor outflow: a review. Exp Eye Res. 2017;158:94-111.

66. Yucel YH, Johnston MG, Ly T, Patel M, Drake B, Gumus E, et al. Identification of lymphatics in the ciliary body of the human eye: a novel "uveolymphatic" outflow pathway. Exp Eye Res. 2009;89:810-9.

67. Tam AL, Gupta N, Zhang Z, Yucel YH. Latanoprost stimulates ocular lymphatic drainage: an in vivo nanotracer study. Transl Vis Sci Technol. 2013;2:3.

68. Da Mesquita S, Louveau A, Vaccari A, Smirnov I, Cornelison $\mathrm{RC}$, Kingsmore KM, et al. Functional aspects of meningeal lymphatics in ageing and Alzheimer's disease. Nature 2018;560:185-91.

69. Rasmussen MK, Mestre H, Nedergaard M. The glymphatic pathway in neurological disorders. Lancet Neurol. 2018;17:1016-24.

70. Mathieu E, Gupta N, Ahari A, Zhou X, Hanna J, Yücel YH. Evidence for cerebrospinal fluid entry into the optic nerve via a glymphatic pathway. Invest Ophthalmol Vis Sci. 2017;58:4784-91.

71. Bair H, Lin CJ, Lai CT, Hsia NY, Tsai YY. Intraocular endoscopy for the evaluation and treatment of hypotony due to a traumatic cyclodialysis: a case report. BMC Ophthalmol. 2020;20:117.

72. Khoo YJ, Abdullah AAH, Yu DY, Morgan WH. Use of trypan blue as an aqueous tracer dye to investigate hypotony where cyclodialysis cleft is suspected. Clin Exp Ophthalmol. 2019;47:904-8.

73. McCartney M, Phagura RS. Delayed bilateral hypertensive crisis with CyPass Micro-stent - The highs and lows. Am J Ophthalmol Case Rep. 2020;18:100635.

74. Grüntzig J, Hollmann F. Lymphatic vessels of the eye-old questions-new insights. Ann Anat-Anatomischer Anz. 2019;221:1-16.

75. Freitas-Neto CA, Costa RA, Kombo N, Freitas T, Oréfice JL, Oréfice $\mathrm{F}$, et al. Subconjunctival indocyanine green identifies lymphatic vessels. JAMA Ophthalmol. 2015;133:102-4.

76. Kim SH, Csaky KG, Wang NS, Lutz RJ. Drug elimination kinetics following subconjunctival injection using dynamic contrast-enhanced magnetic resonance imaging. Pharm Res. 2008;25:512-20.

77. Kronfeld PC. The chemical demonstration of transconjunctival passage of aqueous after antiglaucomatous operations. Am J Ophthalmol. 1952;35:38-45.
78. Addicks EM, Quigley HA, Green WR, Robin AL. Histologic characteristics of filtering blebs in glaucomatous eyes. Arch Ophthalmol. 1983;101:795-8.

79. PowersTP, StewartWC, StromanGA.Ultrastructural features of filtration blebs with different clinical appearances. Ophthalmic Surg Lasers Imag Retina. 1996;27:7904.

80. Teng C, Chi H, Katzin H. Histology and mechanism of filtering operations. Am J Ophthalmol. 1959;47:16-34.

81. Benedikt O. Demonstration of aqueous outflow patterns of normal and glaucomatous human eyes through the injection of fluorescein solution in the anterior chamber (author's transl). Albrecht von Graefes Arch fur Klinische und Experimentelle Ophthalmologie Albrecht von Graefe's Arch Clin Exp Ophthalmol. 1976;199:45-67.

82. Aspelund A, Robciuc MR, Karaman S, Makinen T, Alitalo K. Lymphatic system in cardiovascular medicine. Circulation Res. 2016;118:515-30.

83. Yu D-Y, Morgan WH, Sun X, Su E-N, Cringle SJ, Paula KY, et al. The critical role of the conjunctiva in glaucoma filtration surgery. Prog Retinal Eye Res. 2009;28:303-28.

84. Gong P, Yu DY, Wang Q, Yu PK, Karnowski K, Heisler M, et al. Label-free volumetric imaging of conjunctival collecting lymphatics ex vivo by optical coherence tomography lymphangiography. J Biophotonics. 2018;11:e201800070.

85. Khoo YJ, Abdullah AA, Yu DY, Morgan WH. Use of trypan blue to assess lymphatic function following trabeculectomy. Clin Exp Ophthalmol. 2019;47:892-7.

86. Schroedl F, Kaser-Eichberger A, Schlereth SL, Bock F, Regenfuss B, Reitsamer HA, et al. Consensus statement on the immunohistochemical detection of ocular lymphatic vessels. Investigative Ophthalmol Vis Sci. 2014;55:6440-2.

87. Akiyama G, Saraswathy S, Bogarin T, Pan X, Barron E, Wong TT, et al. Functional, structural, and molecular identification of lymphatic outflow from subconjunctival blebs. Exp Eye Res. 2020:108049. https://doi.org/10.1016/j.exer.2020. 108049.

88. Choi I, Chung HK, Ramu S, Lee HN, Kim KE, Lee S, et al. Visualization of lymphatic vessels by Prox1-promoter directed GFP reporter in a bacterial artificial chromosome-based transgenic mouse. Blood. 2011;117:362-5.

89. Jung E, Gardner D, Choi D, Park E, Seong YJ, Yang S, et al. Development and characterization of a novel Prox1-EGFP lymphatic and Schlemm's canal reporter rat. Sci Rep. 2017;7:1-11.

90. Hong M, Jung E, Yang S, Jung W, Seong YJ, Park E, et al. Efficient assessment of developmental, surgical and pathological lymphangiogenesis using a lymphatic reporter mouse and its embryonic stem cells. PloS ONE. 2016;11:e0157126.

91. Choi I, Lee S, Kyoung Chung H, Suk Lee Y, Eui Kim K, Choi D, et al. 9-cis retinoic acid promotes lymphangiogenesis and enhances lymphatic vessel regeneration: therapeutic implications of 9-cis retinoic acid for secondary lymphedema. Circulation. 2012;125:872-82.

92. Wu Y, Seong YJ, Li K, Choi D, Park E, Daghlian GH, et al. Organogenesis and distribution of the ocular lymphatic vessels in the anterior eye. JCI insight. 2020;5:e135121.

93. Heloterä H, Alitalo K. The VEGF family, the inside story. Cell. 2007;130:591-2.

94. Joukov V, Kumar V, Sorsa T, Arighi E, Weich H, Saksela O, et al. A recombinant mutant vascular endothelial growth factor-C that has lost vascular endothelial growth factor receptor-2 binding, activation, and vascular permeability activities. J Biol Chem. 1998;273:6599-602. 
95. Singh D, Singh RSJ, Singh K, Singh SK, Singh IR, Singh R, et al. The conjunctival lymphatic system. Ann Ophthalmol. 2003;35:99-104.

96. Bouhenni RA, Al Jadaan I, Rassavong H, Al Shahwan S, Al Katan H, Dunmire J, et al. Lymphatic and blood vessel density in human conjunctiva after glaucoma filtration surgery. J Glaucoma. 2016;25:e35-8.
97. Raghava S, Hammond M, Kompella UB. Periocular routes for retinal drug delivery. Expert Opin Drug Deliv. 2004;1:99-114. 98. Lenzhofer M, Strohmaier C, Hohensinn M, Hitzl W, Sperl P, Gerner $\mathrm{M}$, et al. Longitudinal bleb morphology in anterior segment OCT after minimally invasive transscleral ab interno glaucoma gel microstent implantation. Acta Ophthalmol. 2019;97: e231-7. 